\title{
Depression outcomes among Chinese internal migrant workers: Comparisons among subgroups of internal migrant workers
}

\author{
Mi Zhou ${ }^{a}$ and Liuyin Tong ${ }^{b}$ \\ School of Economics, Shenyang Agricultural University, Shenyang 110161, China \\ azhoumisyau@163.com, b1531968151@qq.com
}

Keywords: Depression outcomes, Migrant workers, China.

\begin{abstract}
Migrant workers (MWS) are a large population (277 million by the end of 2015) in China, to date, very limited studies have focused on mental health of MWS especially with a nationally representative database. This study aims to provide insight into the distribution of depression outcomes of migrants in China and identify relatively more vulnerable groups. To achieve this, we used China Family Panel Studies (CFPS) database containing 5913 observations, spanning 25 provinces in China conducted in 2012. Our results indicated that the distribution of depression, estimated with the Center for Epidemiologic Studies Depression Scale (CES-D), was uneven among sub-groups of MWS. The findings showed that MWS were more susceptible to depression than adults with a city hukou. Economic status, education levels, and self-rated health had negative relationships with the depression outcomes among MWS. Otherwise, Migrants that were male performed better in depression than those female. Using the results, this research can provide references and help for government decision making for raising the depression outcomes among MWS.
\end{abstract}

\section{Introduction}

China has been experiencing a fast development of urbanization, leading to the largest migration in history. According to Statistical Bulletin of the People's Republic of China on the 2015 National Economy and Social Development, China's migrant population had reached 277 million by the end of 2015.

In China, internal migration occurs without a change in hukou policy, established in the late 1950s. This policy, initially for industrialization construction, now continues exist, which classifies China's labor force into two distinct segments, one composed of urban workers and the other of rural workers. A person's hukou defines access to employment, housing, social welfare, and educational opportunities [1].

A search yielded 48 cross-sectional surveys and seven surveys (to June 2012) in China indicated that the pooled psychological symptom scores of migrant workers (MWS) were statistically higher than those of norms from general population and those of urban counterpart [2]. And depression is a serious but common mental illness, with an estimated $14.3 \%$ of deaths worldwide, or approximately 8 million deaths each year, attributable to mental disorders [3].

Previous researches have reported that the distribution of depression among different sub-groups of MWS is uneven. But most of these studies were based on limited sampling regions (data were often collected in a specific cities or provinces), moderate sample size (with less than 5000 respondents). Thus, there is an urgent need for a more comprehensive research based on nationally representative data.

The overall goal of this paper is to fill this gap by using the 2012 China Family Panel Studies (CFPS) survey that covers 5913 respondents from 25 Chinese provinces, and build a repository of depression outcomes for MWS in China. In this way, we hope that the results can be used as a decision making tool to help governments target their investments and develop policies which will be aimed at improving the depression outcomes of this vulnerable group in China. Furthermore, we also hope this tool can be employed to help decide which subgroups of MWS need particular special attention. 


\section{Data and Measures}

\subsection{Data.}

CFPS is a nationally representative longitudinal survey that was launched from 2010 biennially by the Institute of Social Science Survey (ISSS) of Peking University. In this paper, we use data from the second wave of CFPS conducted in 2012. Each wave covers about 57000 individuals and 15000 households in 25 provinces of China (all provinces with the exception of Hong Kong, Xinjiang, Macao, Qinghai, Inner Mongolia, Hainan and Ningxia). CFPS focuses on a range of topics related to economic activities, education outcomes, migration and health and family dynamics. This survey also contains three modules: The individual-, family- and community-level in contemporary China. The data for this report come from CFPS (2012)-adults database.

CFPS follows a "Probability-Proportional-to-Size" (PPS) sampling method with three-stage stratification. At the first stage, 162 county-level units were randomly selected. Then, in the same way, 640 village-level units were chosen. Finally, according to the study's systematic sampling protocol, 6317 households from village-level units were selected. All members of each household attended this survey apart from those who were not at home during this investigation.

\subsection{Measures.}

The 2012 wave of the CFPS includes the standard 20 questions of the Center for Epidemiologic Studies Depression Scale (CES-D), which is one of the mostly widely used self-rated tests on the respondents' mental health status. The CES-D test contains 20 items which include 16 negative feelings and 4 positive feelings. For each item, according to the frequency within one week, respondents need to choose from 4 possible answers (table 2):"rarely (less than 1 day),",'Little (1-2 day)","Occasionally (3-4 day)" and "Most (5-7 day)". Correspondingly, each answer is related to an index value of $0,1,2,3$ for negative feelings and 3,2,1,0 for positive feelings, which are added in order to calculate an overall CES-D score ranging from 0 to 60. In this study, if each item in CES-D was not available, we could not calculate the depression score.

In this paper, to make the analysis easier and facilitate the comparison of the degree of variance between different sub-groups, we normalized depression scores using the mean and the standard deviation. Therefore, estimated results were expressed in standard deviation.

\subsection{This Study's Sub-sample.}

The main sample in this paper was a sample of MWS counted 5913, while, at the same time, we also used a sample included 28066 adults which involved farmers, MWS and citizens.

To be eligible for the study, MWS had to satisfy three inclusion criteria: 1) they had a rural hukou; 2) they must have been 18 years or older; 3) they were residing in urban areas at least 6 months per year. We distinguished farmers as those adults who had a rural hukou and engaged in agricultural production. We also defined citizens as those people who were the legitimate citizens living in urban areas, having a valid household registration of the city, and engaged in non-agricultural production with non-rural hukou.

For the convenience of comparing depression outcomes of MWS from different sub-samples, we classified the sample into sub-groups by the characteristics of individual, income and human capital accumulation. Specifically, gender was used to represented individual characteristics, and the income situation consisted of household income and hourly wage, and human capital accumulation status involved education levels and physical health. In particular, self-rated health was measured by asking MWS one question: in general, how do you think your health (not good, moderate, good, very good and excellent).

\section{Results}

We analysis the results with the figure below including a series of sub-graphs. The higher the score, the stronger the depression. 


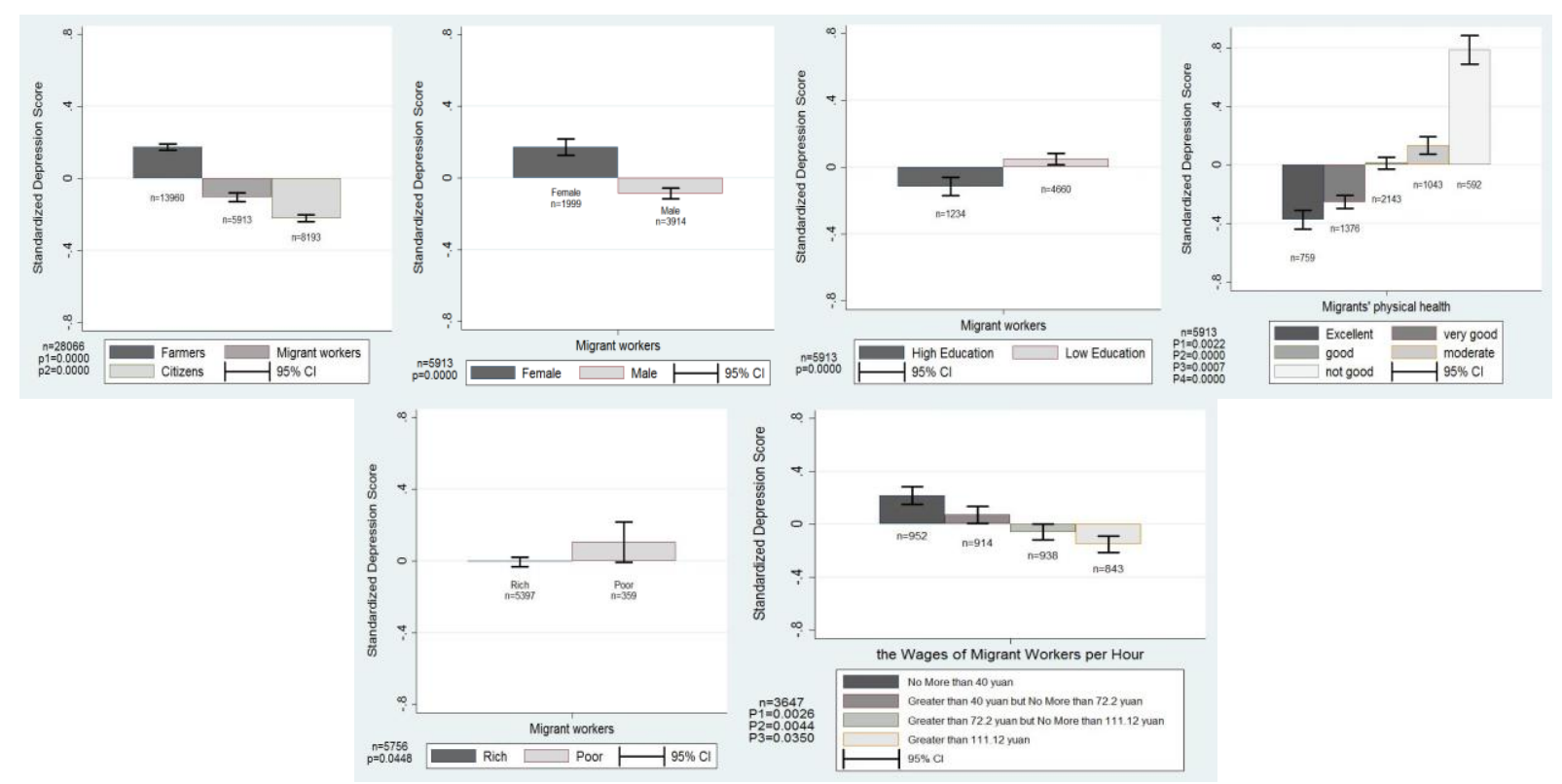

Fig. Depression outcomes among different sub-groups

Depression outcomes of farmers, migrant workers and citizens. We begin our analysis with the overall depression level of MWS. Among the 28066 adults, $21.1 \%$ of them were MWS with $49.7 \%$ farmers and 29.2\% citizens. Comparing the depression performance among adults, the results showed that the standardized depression scores of migrant worker were 0.12 standard deviations higher than those of citizens with the difference significant at the 1 percent level, and 0.28 standard deviations lower than those of farmers with the same level of difference at the 1 percent.

Among 5913 MWS, 66.19\% was males and 33.81\% was female. The standard deviations between the two groups were 0.26 , but this gap was statistically significant at the 1 percent level of significance.

As for depression outcomes among different levels of human capital of MWS, first, We defined the high education as those who had completed nine years of compulsory education, the remnant as the low education. Our exploratory analysis revealed that a relatively identifiable difference of standardized depression scores at the 1 percent level between the two sub-groups was drawn.

Second, concerning physical health, $12.84 \%$ MWS reported excellent health, and $23.27 \%$ reported very good health, $36.24 \%$ reported good health, $17.64 \%$ reported moderate health, and $10.01 \%$ reported bad health. As for the depression scores, they were rising in accordance with the above order with the standard deviations between each other being $0.12,0.27,0.12$ and 0.65 , and the differences between those all groups were significant at the 1 percent level.

For depression outcomes among different income levels of MWS. On the one hand, according to China's poverty line in 2011, we divided MWS into two groups: a (relatively) poor group (359 families) and a (relatively) rich group (5397 families) with 157 missing income values. The results showed that the standardized depression scores of the poor were higher than those of the rich-although the size of gap was relatively small.

On the other hand, we also defined hourly wage as another indicator of income among MWS. They were divided into four roughly equal sub-groups according to the distribution of hourly wage of respondents. We found that mental health was positively associated with level of hourly income. As the figure above showed, standardized depression scores declined with the increasing of hourly income. MWS who earned low hourly income fared worse in psychological well-being than those who earned high hourly income. Specifically, the standard deviation of standardized depression scores between the top quartile of hourly income and the bottom was as significant as 0.4 at the 1 percent level. 


\section{Discussion and Conclusion}

To the best of our knowledge, this is the first systematic study among Chinese MWS of the distribution of depression outcomes with much larger sample size (and broader scope). As a result, we successfully build a repository of depression outcomes for various type of MWS across the country.

The findings show that MWS performed worse in depression outcomes than adults with a city hukou, and the distribution of depression outcomes among MWS is uneven in different sub-group. Economic status, education levels, and self-rated health have negative relationships with the depression outcomes among MWS. Otherwise, Migrants that are male perform better in depression than those are female. And the difference of standardized score between these sub-groups showed the most significant.

From a policy perspective, if China's government wants to raise depression outcomes among MWS, awareness of mental health problem and uneven distribution among this group is the first step. Otherwise, considering the overall worse performances of MWS than citizens who have a city hukou in depression outcomes, the fundamental changes to the restrictive hukou system may be the most effective way to improve the mental health of MWS in general. Another potential direction of policy change would be to focus on the raise of minimum wage standard, improving education levels and providing effective and affordable medical services for MWS. And for improving education levels, China is time to provide free high school education.

\section{References}

[1]. Chan, K.W. Post-Mao China: A Two-Class Urban Society in the Making. International Journal of Urban and Regional Research. Vol. 20 (1996) No. 1, p. 134-150.

[2]. Zhong, B.-L., et al. Prevalence of psychological symptoms in contemporary Chinese rural- tourban migrant workers: an exploratory meta-analysis of observational studies using the SCL-90-R. Social Psychiatry and Psychiatric Epidemiology. Vol. 48 (2013) No. 10, p. 1569-1581.

[3]. Walker, E.R., R.E. McGee, and B.G. Druss. Mortality in mental disorders and global disease burden implications: A Systematic Review and Meta-analysis. Jama Psychiatry. Vol. 72 (2015) No. 4, p. 334-341. 